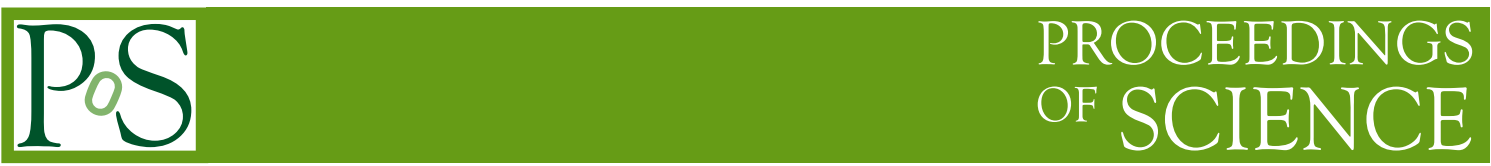

\title{
Gemini Operations for Multi-Messenger Astronomy
}

\author{
Bryan W. Miller*, Andy Adamson, John Blakeslee, Andrew Stephens, Joanna \\ Thomas-Osip, and Arturo Núñez \\ Gemini Observatory \\ E-mail: bmiller@gemini.edu
}

\begin{abstract}
Gemini Observatory will be an important facility for following up time-domain discoveries in the multi-messenger era. Gemini has a variety of time allocation processes in order to accommodate a broad range of project needs and timescales. Time is allocated by regular participant TACs, a common large program TAC, and by proposer peer review for "fast-turnaround" proposals. Queue observing allows Gemini to easily execute target-of-opportunity (ToO) observations and this capability will be very important for transient follow-up. Instrumentation includes optical and near-infrared imagers and spectrographs at both sites. New facility instruments and systems are under development including GHOST (high-resolution optical spectrograph), SCORPIO (the broad-wavelength follow-up workhorse), and a new multi-conjugate AO system for Gemini North. Visitor instruments are also highly encouraged. All new facility instruments will be delivered with data reduction pipelines and the data are delivered via a cloud-based science archive. Finally, we summarize planned changes to our operations software to handle the expected increased volume of $\mathrm{ToO}$ triggers and to incorporate Gemini into the developing time-domain follow-up infrastructure. These changes will include new interfaces, more programmatic access, a real-time scheduler, and automated data reduction.
\end{abstract}

The New Era of Multi-Messenger Astrophysics - Asterics2019

25 - 29 March, 2019

Groningen, The Netherlands

${ }^{*}$ Speaker. 


\section{Introduction}

Gemini Observatory is an international partnership that operates twin 8-meter optical-infrared telescopes with coverage of the entire sky from Cerro Pachón in northern Chile and Maunakea on Hawaii's Big Island. The full participants (United States, Canada, Brazil, Argentina, and the Republic of Korea) have full access to all facilities and modes. Along with hosts Chile and the University of Hawaii, the participants run National Gemini Offices (NGOs) that help with user support. Gemini also has limited-term participants that have access to limited modes and time is exchanged with Subaru and the Canada-France-Hawaii Telescope. The vast majority the time is used for some form of queue observing in which staff observers execute observations when the conditions are appropriate. This makes Gemini particularly good at responding rapidly to timedomain and multi-messenger events. This contribution describes Gemini's current operations and changes that are underway to make operations even more flexible for the era of multi-messenger astrophysics.

\section{Proposal Processes and Instrumentation}

Gemini's flexibility starts with the proposal. There are a variety of proposal opportunities for experiments of different sizes and timescales. As seen in Table 1, different types of proposals are due with different frequencies and are used to allocate different fractions of the telescope time. National time allocation committees (TACs) for the full participants allocate most of the time on a semester schedule. A single large and long program TAC that meets once a year allocates about $20 \%$ of the time for larger than normal projects. Fast turnaround proposals are due once a month and they are ranked by representatives of the teams that submit them[3]. Fast turnaround is useful for pilot projects, finishing a sample, or anything that is needed within a few months.

\begin{tabular}{llcl}
\hline \hline Proposal Process & Frequency & Fraction of Time & Project Duration \\
\hline Semester Queue/Classical & Every 6 months & $\sim 70 \%$ & $6-12$ months \\
Large and Long Programs & Every year & $20 \%$ & Up to 6 semesters \\
Fast Turnaround & Every month & $10 \%$ & 3 months \\
Director's Time and Poor Weather & Any time & $<5 \%$ & Weeks to months \\
Demo Science, System Verification & Special cases & & Weeks to months \\
\hline
\end{tabular}

Table 1: Gemini proposal options.

The instrument options also contribute to flexible operations. Each site has four facility instruments plus an adaptive optics (AO) system. Three instruments plus the AO system can be mounted simultaneously on the Cassegrain instrument support structure. Switching instruments involves merely moving the tertiary fold mirror and takes less than a minute. Both telescopes have optical and near-infrared imagers and spectrographs with MOS and IFU capabilities. Gemini South has the world's only operational multi-conjugate AO-system (MCAO) behind a five laser guide-star constellation. Gemini North's AO facility is the single laser guide-star Altair system, but there are plans to upgrade this (see below). Many visitor instruments such as Phoenix, TEXES, IGRINS, 'Alopeke/Zorro, MAROON X, and GIRMOS also take advantage of Gemini's large collecting area and low thermal emissivity. 
Several new facility instruments are under development. GHOST is under construction and will be a two-object, high-resolution $(R=50,000-75,000)$ optical cross-dispersed spectrograph. SCORPIO will be an eight-channel $0.4-2.4 \mu \mathrm{m}$ imager and longslit spectrograph designed for transient follow-up. It is currently in the design phase. Finally, the Republic of Korea is planning to build a new version of the popular IGRINS $R=45,000$ near-infrared cross-dispersed spectrograph as a Gemini facility instrument. All new facility instruments will be delivered with data reduction software that will run within the Python-based DRAGONS pipeline environment.

\section{The Future of Time-Domain and MMA Follow-up}

A high priority of the U.S. National Science Foundation (NSF) is to maximize the science return from the major facilities that it has funded or operates such as LIGO, IceCube, LSST, Gemini, CTIO, and SOAR. The U.S. National Research Council report Optimizing the U.S. Ground-Based Optical and Infrared Astronomy System [1] recommends that NSF facilities coordinate to optimize LSST follow-up. The same capabilities are needed for MMA follow-up. The Gemini Board's guidelines state that "Gemini will strive to be the best observatory in the world for the execution of flexible, innovative, and efficient science programs."

Target of Opportunity observations already make up 20-25\% of the time used in Gemini's highest ranking band. This results in at most 1-2 new requests per night. Many more, as would be expected with $\sim 10^{7}$ LSST alerts per night, will likely overwhelm the current manual scheduling process and the observers. At classically-scheduled telescopes such as SOAR and the Blanco 4meter more ToOs will be disruptive to visitor programs. At the moment, coordinating observations between different institutions such as Gemini, HST, Chandra, or the VLA is done via email, a process that is inefficient and error prone. A new solution is needed.

The solution under development is a system that dynamically turns alerts into data and is diagramed in Figure 1. This system consists of: 1) brokers that will aggregate, classify, and filter alerts; 2) Target Observation Managers (TOMs) that are used by science teams for prioritizing targets and managing observations and data; and 3) observatory interfaces, schedulers, facilities, along with data reduction software and science archives.

Several groups are developing brokers for aggregating the alerts with catalogs and classifying objects. NOAO's ANTARES (antares.noao.edu) is now in alpha release and users can define filters for Zwicky Transient Facility (ZTF) alerts. A group in Chile is developing AleRCE (alerce.science) and the UK's Lasair (lasair.roe.ac.uk) is also processing ZTF alerts[2].

A Target Observation Manager (TOM) is a tool used by a science team to match targets of interest with the telescopes to which the team has access, to submit and track observations, and to organize and visualize the data that is returned. Several of these are currently in use by teams studying supernovae, exoplanets, near-earth objects, AGN, and microlensing events[4]. Until now each team has had to develop its own custom software. Fortunately, some of the astronomers at Las Cumbres Observatory have experience with the early TOMs and have identified common required functionality. These features have now been made part of a TOM Toolkit (tomtoolkit.github.io) that will make it easier for any team to develop a TOM. Gemini has developed a plugin for the TOM Toolkit so that ToOs can be triggered using the existing limited API (see Figure 2). A video showing some of the TOM's functions and how triggering Gemini observations works can be found 


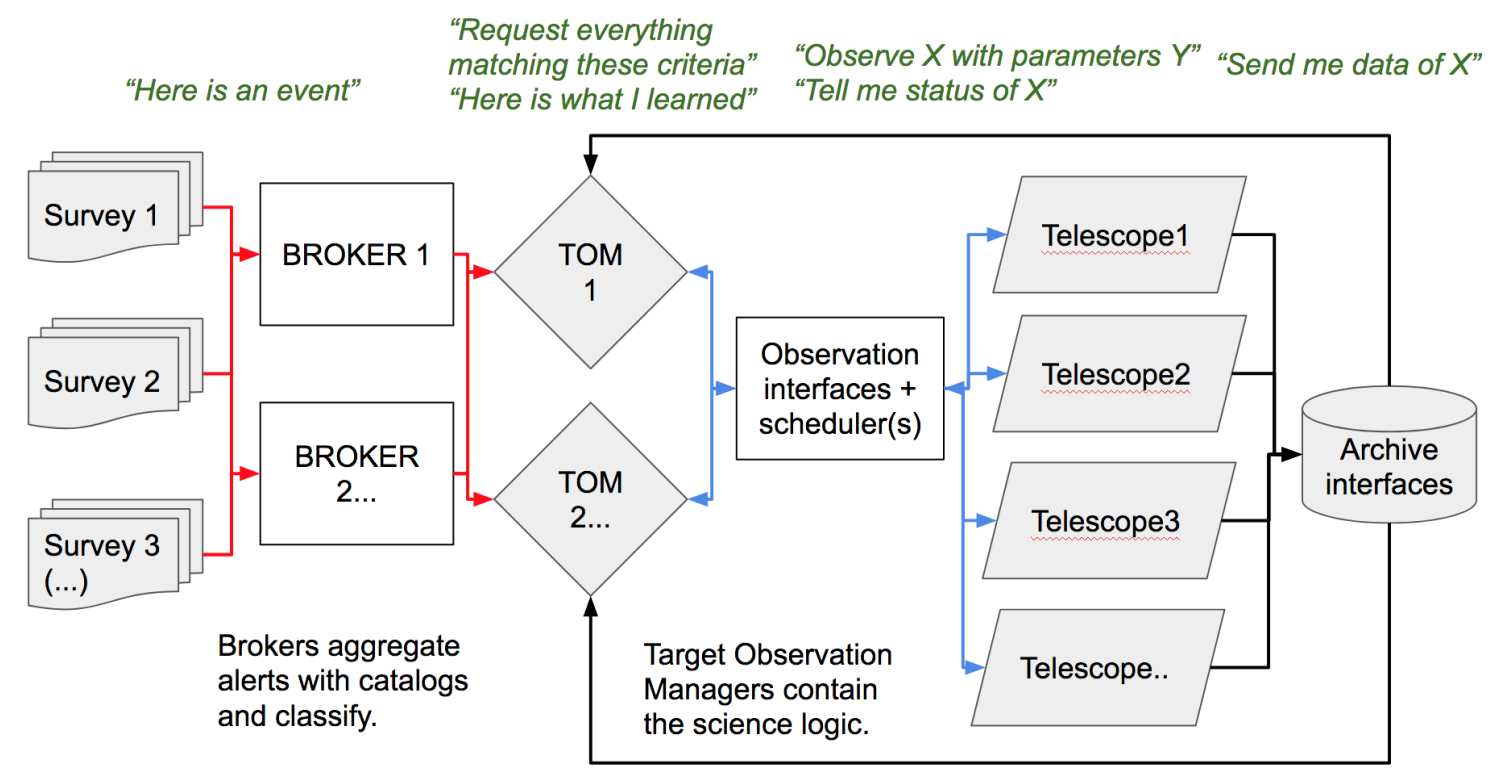

Figure 1: A network flow diagram for transient follow-up. AEON includes observation interfaces, schedulers, telescopes (Gemini, SOAR, Las Cumbres, Blanco, etc) and their associated data reduction and archives (Figure concept: Rachel Street, Las Cumbres Observatory).

on YouTube ${ }^{1}$. The TOM Toolkit is now being used to follow-up LIGO events on Las Cumbres and Gemini.

TOMs will send observation requests to the observatories programmatically via application programming interfaces (APIs). Then the observatories need to execute them efficiently and on the appropriate timescale. Since very rare or rapidly evolving events may need to be observed quickly, dynamical scheduling is needed that can change the observing plan in real time and without significant human interaction. Many projects also need access to multiple facilities. For example, the study of the explosions resulting from binary neutron-star mergers detected with LIGO requires imaging surveys followed by spectroscopic characterization. Supernovae studies may need different aperture telescopes depending on the brightness of the source, or they may use smaller telescope for classification followed by larger facilities for higher-resolution observations. Therefore, it would be more efficient if investigators could request time on multiple facilities with a single proposal.

One effort to provide these capabilities is the Astronomical Event Observatory Network (AEON), an initiative by NOAO that includes Las Cumbres, SOAR, and Gemini. The goals of this effort are to develop the necessary APIs, build on the Las Cumbres experience to provide dynamic scheduling capability for SOAR and Gemini, and coordinate data reduction and archiving efforts. So far the Las Cumbres interfaces have been updated to include the SOAR Goodman spectrograph, Goodman scripting capabilities have been implemented, and observing plans from the Las Cumbres scheduler have been executed on SOAR on engineering nights. Some shared-risk science use is planned for semester 2019B. New proposal options for the network are also being considered.

\footnotetext{
${ }^{1} \mathrm{https}: / /$ youtu.be/PC_5kmSdZBU
} 
을 Toolkit Home Targets Alerts Observations Data Users

User 1 Logout

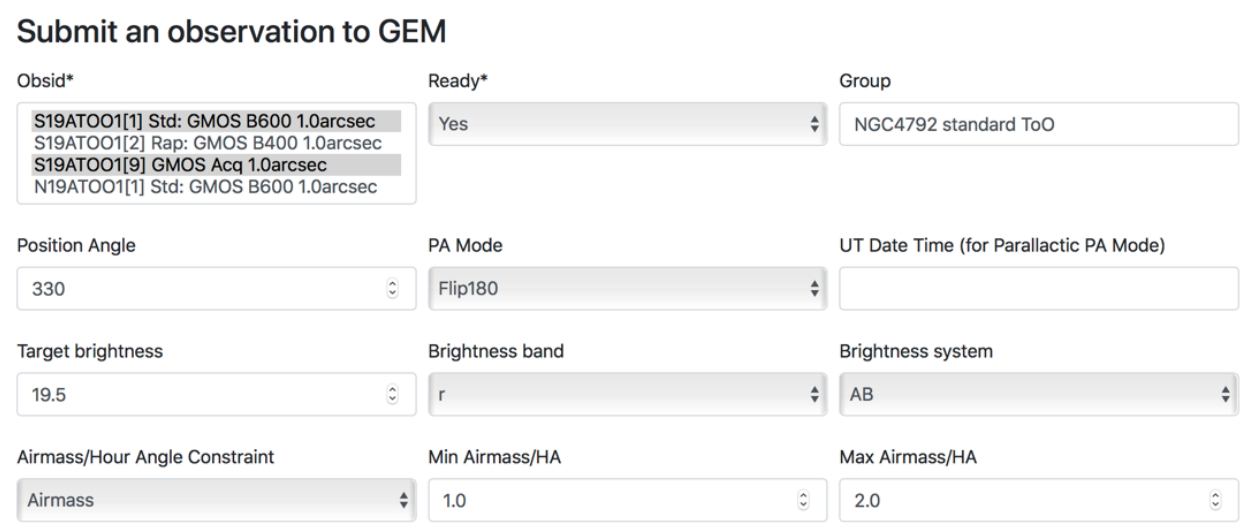

Figure 2: Part of the user interface for defining a Gemini observation with a Target Observation Manager (TOM) created with the TOM Toolkit. Multiple observation templates can be selected. The software will also find a guide star and then send the information to the Gemini observing system.

Gemini will support AEON as part of the ongoing Observatory Control System (OCS) Upgrades Program and new supplemental funding from the NSF for a major project called Gemini in the Era of Multi-Messenger Astronomy (GEMMA). The Gemini observing system needs to be updated to make it easier to use, to include new features for automated scheduling, and to make it API driven. The GEMMA funds are being used for a MMA-related outreach program, a new MCAO system for Gemini North, new real-time computers for the MCAO systems, and the realtime queue scheduler and data reduction capabilities to support AEON. The data reduction work involves transitioning the old IRAF-based code to the new DRAGONS (Python) environment and is being done in coordination with SOAR.

\section{Summary}

Gemini is re-imagining its observing system in order to provide more dynamic, flexible capabilities that will allow it to be a productive member of a time-domain and multi-messenger followup network. The changes are designed to benefit all users and the new projects will also add significant new instrumentation capabilities, positioning Gemini well for the 2020s and beyond.

\section{References}

[1] D. M. Elmegreen, et al. Optimizing the U.S. Ground-Based Optical and Infrared Astronomy System, National Academies Press, Washington D.C. 2015

[2] J. Lightfoot, 2019, in these proceedings

[3] R. E. Mason, S. Côté, M. Kissler-Patig, N. A. Levenson, A. Adamson, C. Emmanuel, \& D. Crabtree, D. The Gemini Observatory Fast Turnaround Program, SPIE, 9149, 914910, [astro-ph/14085916]

[4] R. A. Street, M. Bowman, E. S. Saunders, \& T. Boroson, General-Purpose Software for Managing Astronomical Observing Programs in the LSST Era, SPIE, 10707, 1070711, [astro-ph/180609557] 